\title{
Tridimensional Vectorial Modeling of the Heart and Coronary Vessels from the Anatomical Slices of the Korean Visible
}

\section{Human}

\author{
François Uhl J1*, Hautin $\mathrm{R}^{1}$, Park JS ${ }^{2}$, Chung BS ${ }^{3}$, Latremouille $\mathrm{C}^{3}$ \\ and Delmas $\mathrm{V}^{4}$ \\ ${ }^{1}$ URDIA Unit EA 4566, Department of Anatomy, Paris Cité Sorbonne University, \\ France
}

2Department of Anatomy, Dongguk University School of Medicine, Republic of Korea 3Department of Anatomy, Ajou University School of Medicine, Republic of Korea ${ }^{4}$ Department of cardiac surgery - European hospital Georges Pompidou, France

\section{Research Article \\ Volume 1 Issue 3}

Received Date: November 12, 2017

Published Date: December 08, 2017

*Corresponding author: Jean-François UHL, Department of Anatomy, Paris Cité Sorbonne University, France, Tel: 0173007675; E-mail: Jeanfrancois.uhl@gmail.com

\section{Abstract}

The virtual dissection is a remarkable learning tool in anatomy. It is particularly useful in the case of lack of cadavers or if anatomical dissection is impossible due to ethical or religious reasons. The authors present here a 3D reconstruction of the female's heart from the Visible Korean human data, made in the frame of the projects of the UNESCO chair of digital anatomy created recently at the Descartes University.

The manual segmentation of 1640 anatomical slices was achieved with the Winsurf ${ }^{\circledR}$ software producing an interactive 3D vectorial model of the heart and surrounding anatomy. Eighty four anatomical structures were reconstructed, including the heart and its vessels (27 structures), trachea, oesophagus, lungs, aortic arch, superior vena cava, azygos system, inferior vena cava, right and left kidneys, skeletal system (58) structures including: sternum, xiphoid process, clavicles, ribs, costal cartilage, thoracic vertebrae, intervertebral discs, sacrum, hip bones, and femurs) and skin.

The obtained 3D vectorial model was exported in 3D PDF format, producing a true virtual dissection tool through the Acrobat's interface: the anatomical structures can be individually and interactively manipulated as 84 separated 3D objects. 3D labels can be added with the name of each anatomical element. This new computerized virtual dissection table is a simple and powerful learning tool for students and anatomy teachers. It is also the basis of future simulation tools for surgeon's training.

Keywords: Three-dimensional anatomy; Visible Korean human; Heart modeling; Coronary vessels; Virtual reality; 3D reconstruction; Virtual dissection; Surgical simulation; Surgical training 
Abbreviations: VHP: Visible Human Project; CVH: Chinese Visible Human; VCH: Virtual Chinese Human

\section{Introduction}

Sectioned images of the human body are of considerable interest due to their high resolution to build 3D atlases of the human body based on vectorial modeling.

Since the first set of anatomical slices of the US Visible Human Project (VHP; male and female) conducted in the United States [1,2]. Several projects were setup: Visible Korean (male whole body, male head, and female whole body) [3-5]. Chinese Visible Human (CVH; male and female) [6] Virtual Chinese Human ( $\mathrm{VCH}$; male and female) $[7,8]$.

The educational use of these 3D anatomical models of the whole body is of high value thanks to several computer and imaging tools developed recently: browsing software of the anatomical and segmented images [9], virtual dissection with Acrobat ${ }^{\circledR}$ 3D PDF interface [10], or dedicated system with a table like Anatomage ${ }^{\circledR}$ [11], simulation of simple surgical procedures [12], virtual reality mask (dedicated software developed with Samsung Oculus ${ }^{\circledR}$ Gear VR) [13].

\section{Material and Methods}

The subject was a Korean male cadaver aged 33 years of standard body size, $1 \mathrm{~m} 64$ and weight $55 \mathrm{~kg}$ : who died after a lymphoma frozen at $-70^{\circ} \mathrm{C}$ [5]. A cryomacrotome was used for serial-sectioning the whole body from the vertex to the perineum at $0.2 \mathrm{~mm}$ interval and from the perineum to the toes at $1 \mathrm{~mm}$ interval. That produced 5960 anatomical slices of the whole body [5]. For photographing the sectioned surfaces, a Canon ${ }^{\mathrm{TM}}$ EOS-1Ds Mark III ${ }^{\mathrm{TM}}$ was used, equipped with a $\mathrm{Canon}^{\mathrm{TM}} \mathrm{EF} 50 \mathrm{~mm}$ f/1.2L USM lens. The resolution of the resulting images was 5,616 X 3,744 pixels, saved in the TIFF format.

\section{Segmentation and 3D Reconstruction}

Instead of firstly performing segmentation with Photoshop [14] we directly did the segmentation on the anatomical slices of the KVW dataset numbered 1500 to 2500 by using the Winsurf ${ }^{\circledR}$ software [15-17]. The main steps and functions of the software were the following, previously explained in our paper [18] to reconstruct the uro-genital tract of the Korean visible human.

Creation of New 3D Objects: Each 3D object representing an anatomical structure was built separately, identified by placing manually chains of points around the object edges, by this way each distinct anatomical structure was segmented by mapping. Figure 1 shows the software interface, with the available tools on the left upper side of the window to edit or modify the contours. Here the boundaries of the right ventricle were drawn.

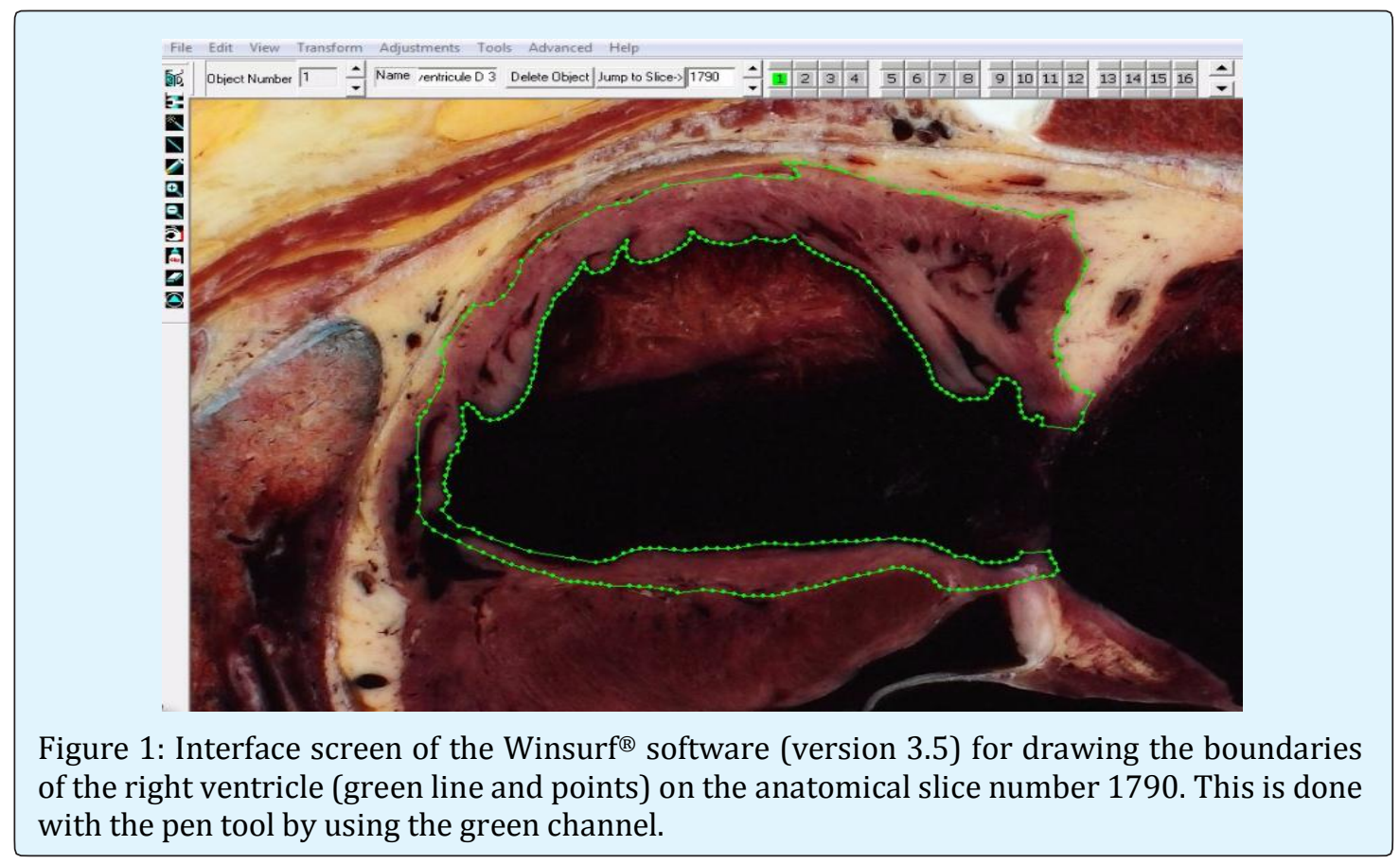

UHL J Francois et al. Tridimensional Vectorial Modeling of the Heart and Coronary Vessels from the Anatomical Slices of the Korean Visible Human. J Human Anat 
Scale measurement of the 3D model: The scale parameters were measured on a slice as well as the slice thickness by a special function of the software. The resulting value was $7000 / 2 / x$, where $x$ represents the jump between slices during display. The same scale parameters 7000/2) are to be used for all anatomical objects.

Creation of 3D objects: 3D object is achieved by outlining them slice by slice (manual segmentation of the objects). Once the contours had been assigned to each serial slice containing the object, vertices were connected using a surfacing routine developed especially for Winsurf ${ }^{\circledR}$ software. This routine computes and refines a best-fit solution for assembling the vertices into a volumetrically optimized 3D object. Figure 2 shows how to use the several color channels to build the arborescence of the coronary veins.

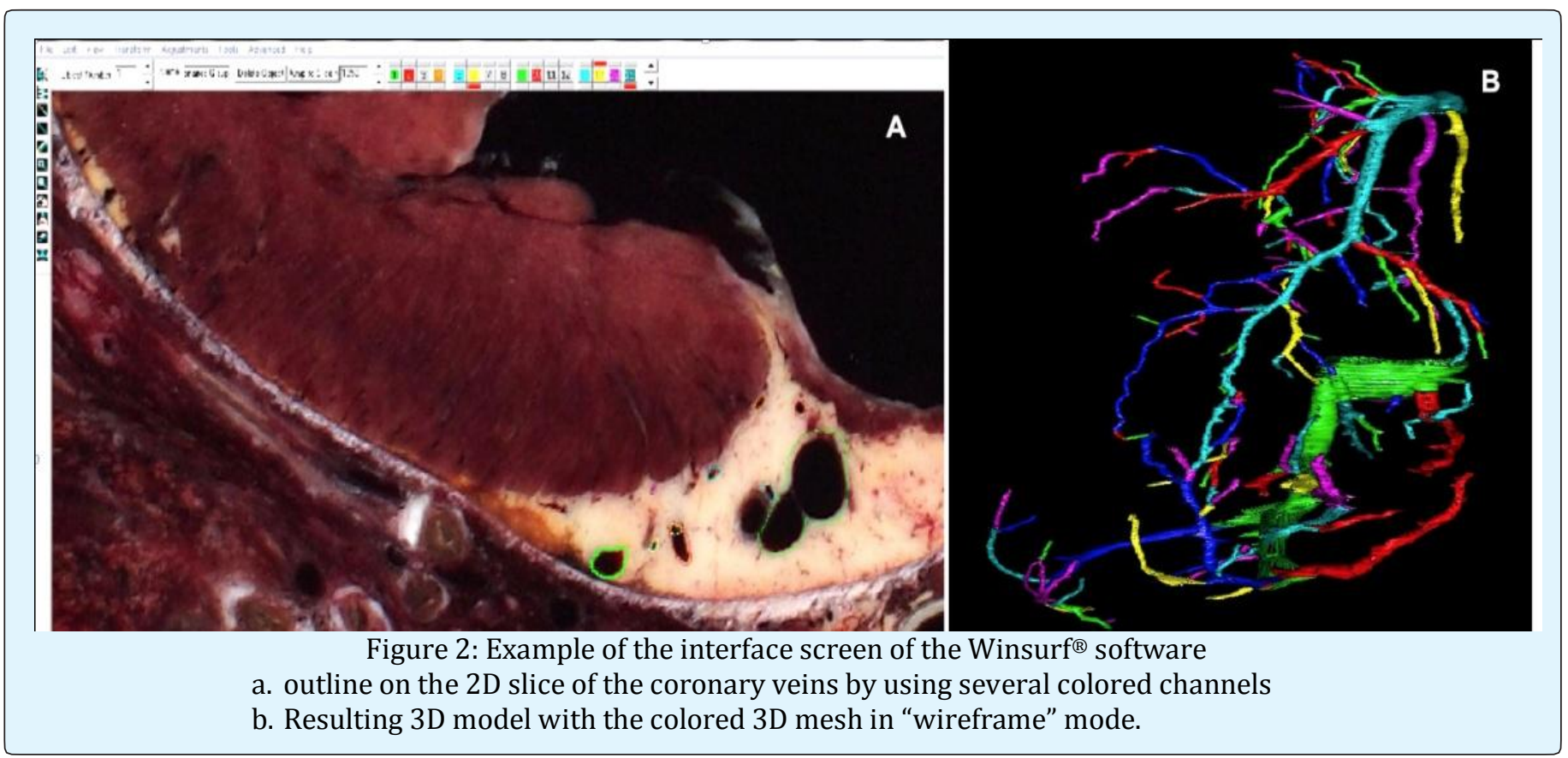

When finished, the whole objects could be gathered by using the "add surface file" option of the software, in order to obtain the final 3D model. In addition, textures could be applied on the objects (particularly the heart cavities and lungs) to obtain a more realistic 3D reconstruction.

\section{Exportation of the 3D Model into 3D PDF Format}

The second step was the export of the whole 3D vectorial model built by Winsurf into 3D PDF format in order to take advantage of the Acrobat interface [10].
Before this exportation, it is important to ensure that every anatomical object has a different RGB color, in order to import it as a separate anatomical element under the Acrobat format. The format conversion was achieved by using a simple function of Adobe Acrobat 3D version 9, in the menu option of the software: "create a pdf file by 3D capture".

Finally, we used Acrobat 3D toolkit software to setup the hierarchical list of the anatomical elements (Figure 3), displaying them in the model tree window located on the left side of the Acrobat window [19,20]. 


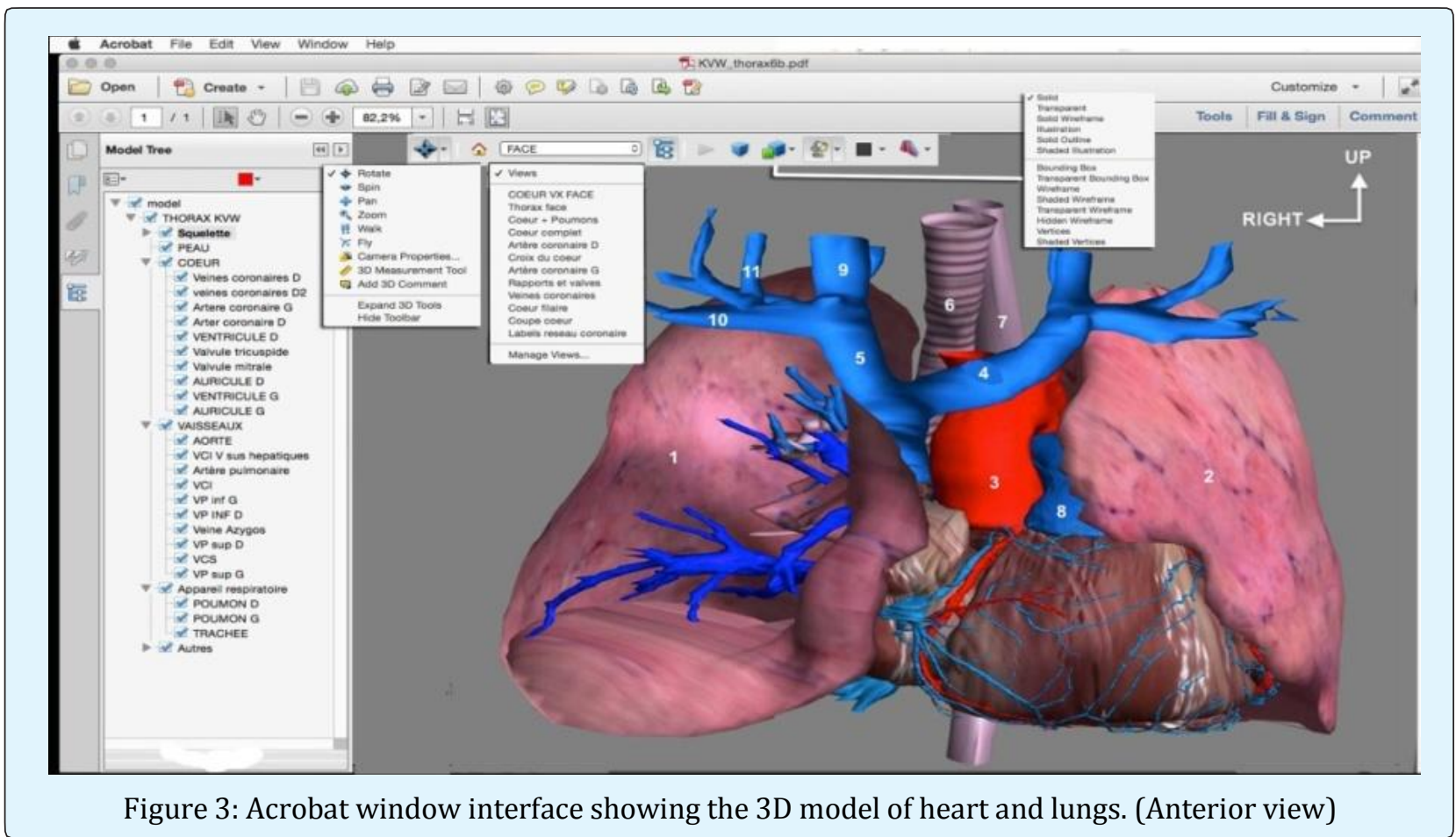

1-Right lung; 2-Left lung; 3-Ascending aorta; 4-Innominate trunk; 5-Right venous jugular-subclavian confluent; 6Trachea; 7-Oesophagus; 8-Trunk of the pulmonary artery; 9-Right internal jugular vein; 10-Right subclavian vein; 11: Right external jugular vein

\section{D interactive display with Acrobat 3D PDF interface}

By clicking the boxes of this list of anatomical structures (model tree) one can make any element visible, hidden or transparent. Other functions of the Acrobat interface are also available in the toolbar located at the top of the display window (Figure 3). Menu of interactive handling functions of the 3D model, choice of lighting effects, background color, 3D rendering options (solid, wireframe, shaded wireframe, transparent wireframe, illustration...), 3D labels, cross section in any plane and "menu" memorizing the display view together with the whole parameters of the 3D anatomical model. All these functionalities will be soon available through a virtual dissection table device (www.anatomieunesco.org/diva3d) extended to the whole body of the male and female.

\section{Results}

83 anatomical structures were segmented to build the whole 3D model: (Table I)
Heart and coronary vessels: right and left ventricle, right and left atrium, mitral valve (with the 2 leaflets), tricuspid valve (with the 3 leaflets), right coronary artery, heart crux, marginal branches, posterior retroventricular artery, posterior interventricular artery, left coronary artery, circumflex artery, anterior interventricular artery, septal branches, diagonal branches, "bissectrix" artery, coronary sinus, anterior interventricular vein, posterior interventricular vein, posterior veins of the left ventricle, Marshall vein, great vein of the heart, middle vein of the heart.

In addition, several surrounding structures were reconstructed: trachea, oesophagus, lungs, aortic arch, superior vena cava, Azygos system, inferior vena cava, right and left kidneys, sternum, xiphoid process, left and right clavicle, ribs, costal cartilage, thoracic vertebrae, intervertebral discs, sacrum, hip bones, femurs, and skin.

The Figure 3 shows the heart with the surrounding mediastinal structures, Heart \& its vessels are shown on (Figures 4 \& 5). 


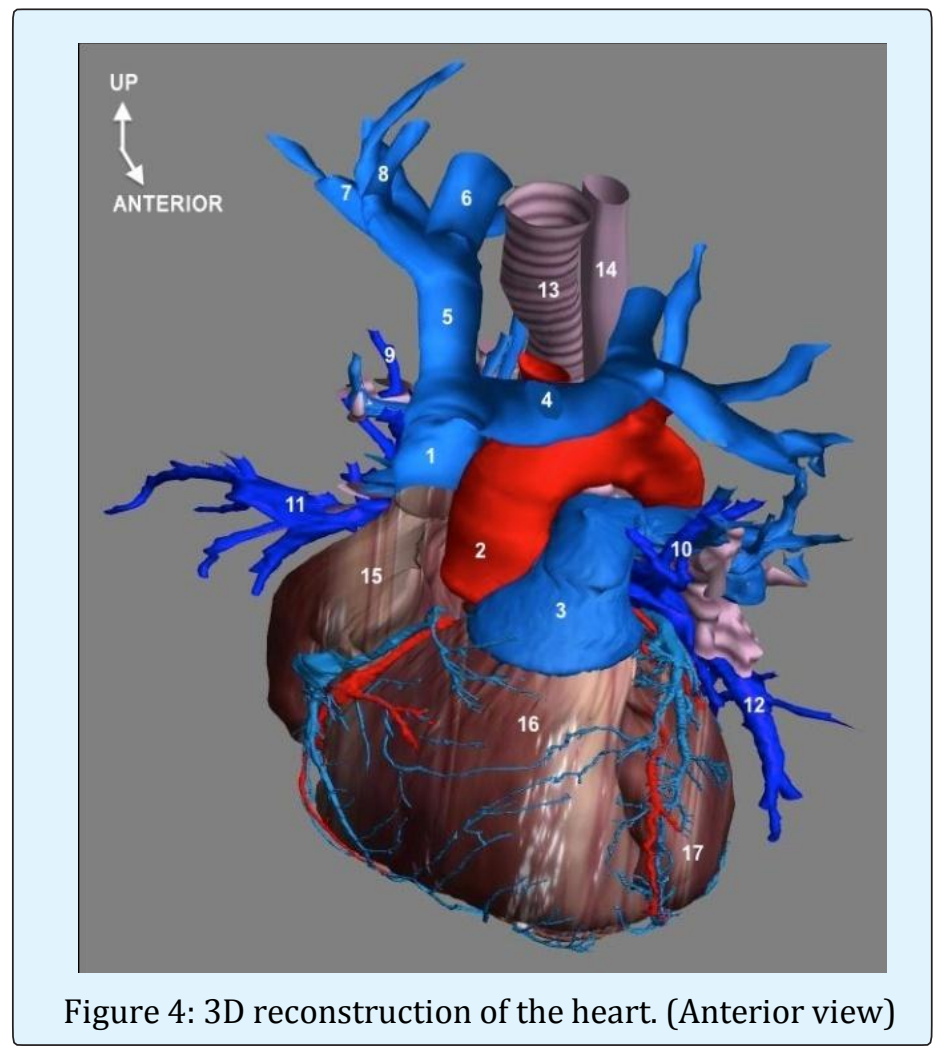

1-Superior vena cava; 2- Ascending aorta; 3- Trunk of the pulmonary artery; 4- Innominate venous trunk; 5- Right venous jugular-subclavian Confluent; 6- Right internal jugular vein; 7- Right subclavian vein; 8- Right external jugular vein; 9Upper right pulmonary vein; 10- Upper left pulmonary vein; 11- Lower right pulmonary vein; 12- Lower left pulmonary vein; 13- Trachea; 14- Oesophagus; 15- Right atrium; 16- Right ventricle; 17- Left ventricle

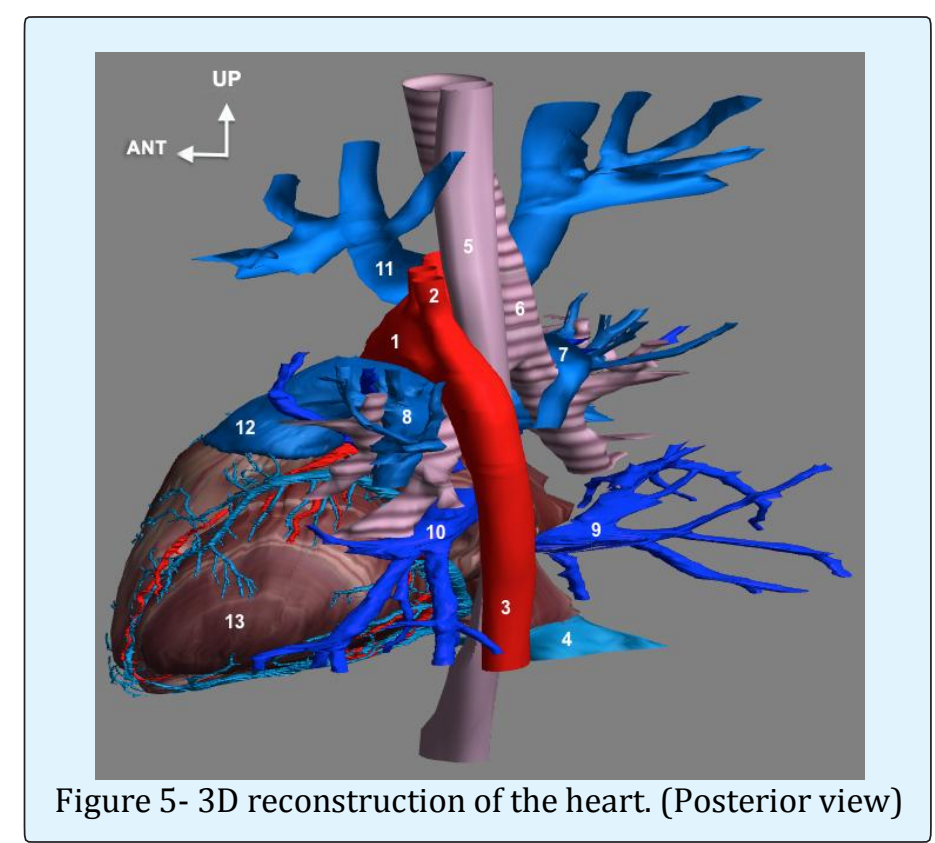

1- Ascending aorta; 2- Left subclavian artery; 3- Thoracic descending aorta; 4- Inferior vena cava; 5- CEsophagus; 6Trachea; 7- Right pulmonary artery; 8- Left pulmonary artery; 9- Lower right pulmonary vein; 10- Lower left pulmonary vein; 11- Innominate trunk; 12 - Trunk of the pulmonary artery; 13- Left ventricle 


\section{Anatomical Description of the Coronary Arteries}

The right coronary artery takes birth in the right anterior sinus of Valsalva at the aortic root. It runs along the right atrio-ventricular groove generally describing a form of "C" with 3 portions- D1, and short horizontal portion, D2, long vertical portion and D3, long horizontal portion.

The junction D1 / D2 is the "genu superius" and D2 / D3 junction at "genu inferius ". Its main report is the tricuspid valve, and thus risks to be harmed during valvular replacements.

It quickly gives birth to the infundibular artery (or conus artery) next to the right atrium, which is one of the first tributaries of the right coronary artery with the artery of the sinusal node. Then marginal tributaries arise throughout the course of the artery, also ensuring vascularization of the right heart.

The right coronary artery ends at the back of the heart, at a special location called the "crux" or cross of the grooves. It gives birth to its terminal branch called posterior inter-ventricular artery, running along the posterior inter-ventricular groove, located at the lower part of the inter-ventricular septum and connecting with the anterior inter-ventricular septal artery and its tributaries.

We also see the posterior retro-ventricular artery trudging through the left atrio-ventricular groove and participating in the vascularization of the lateral wall of the left ventricle.

Finally, septal tributaries arise from this "heart's crux". They are particularly important because they vascularize the sinus node.

The left coronary artery appears in the aortic root at the left anterior sinus of Valsalva where takes birth the common trunk of the left coronary artery.

This short trunk ( 1 to $4 \mathrm{~cm}$ ), passes behind the common trunk of the pulmonary artery at the level of the pulmonary valve.

It is quickly divided into circumflex artery and left anterior interventricular artery:

- The circumflex artery starts from the common trunk of the left coronary artery and runs at the posterior surface of the heart along the left atrio-ventricular groove. It gives on average 2 to 4 marginal branches of the left heart border and ends next to the heart's crux. Its main report is the mitral valve and thus risks to be harmed during valvular replacement.

- The anterior interventricular artery arises from the common trunk of the left coronary artery and runs along the anterior inter-ventricular sulcus to the heart surface. It divides into 2 types of collateral tributaries: septal tributaries, perpendicular, which sink into the septum and diagonal tributaries, which stay at the surface of the heart.

Please note that between the anterior interventricular artery and the circumflex artery, the artery that bisects, depending on its orientation, is attached to one or the other for its territory. Note also the absence of anastomosis between the posterior and anterior descending arteries.

\section{Anatomy of the Venous Network of the Heart}

The coronary sinus is a venous trunk located to the back of the heart in the left atrio-ventricular groove draining almost entire cardiac venous system.

It opens into the right atrium between the septal leaflet of the tricuspid valve and the orifice of the inferior vena cava. This makes one of Koch's triangle boundaries.

The most important veins of the heart shown in this 3D model:

1. The great cardiac vein is the main vein of the heart. It arises at the apex of the heart, runs along the anterior inter-ventricular groove close to the anterior interventricular artery, then continues in the left atrioventricular groove where it becomes satellite of circumflex artery before ending into the coronary sinus. So the great heart's vein drains the anterior and left side of the heart.

2. The middle cardiac vein is continuous with the great vein of the heart. It runs along the posterior interventricular groove and drains into the coronary sinus in the back and bottom of the heart.

3. The small cardiac vein is particularly interesting because it is the only cardiac vein ending directly in the right atrium and not into the coronary sinus. It is the companion vein of the right coronary artery. 


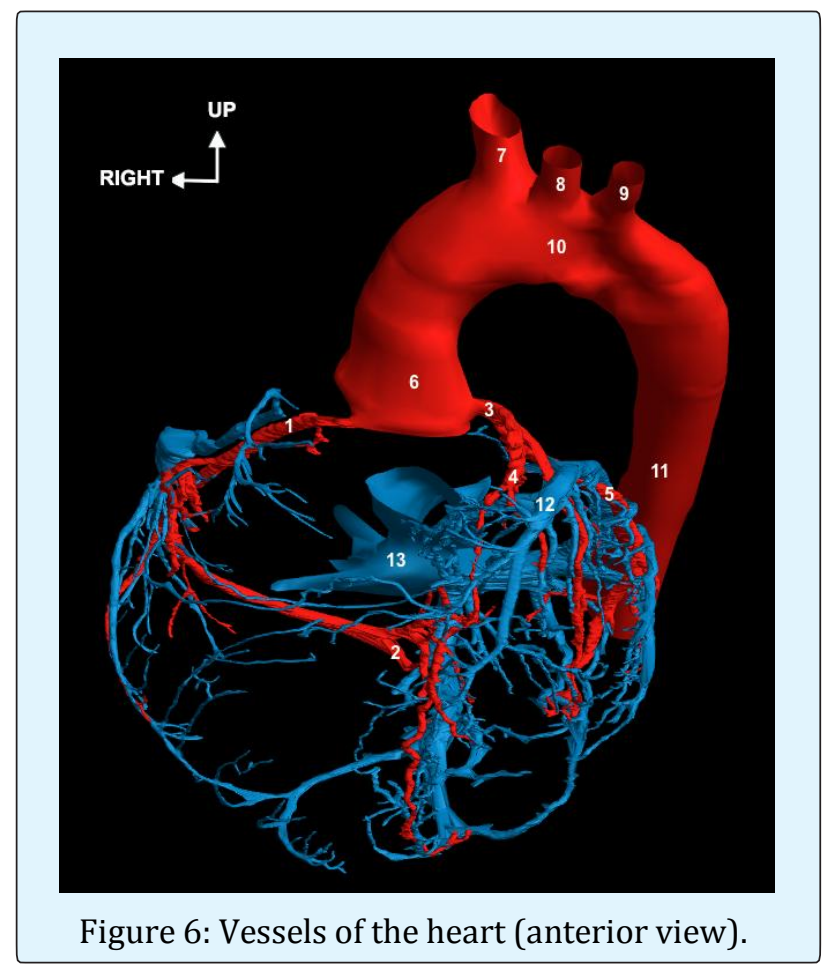

1-Right coronary artery; 2-Right marginal artery; 3-Left coronary artery; 4-Anterior interventricular artery; 5-Circumflex artery; 6-Ascending aorta; 7-Brachio-cephalic arterial trunk; 8-Left common carotid artery; 9-Left subclavian artery; 10Aortic arch; 11-Thoracic descending aorta; 12-Great cardiac vein; 13-Inferior vena cava

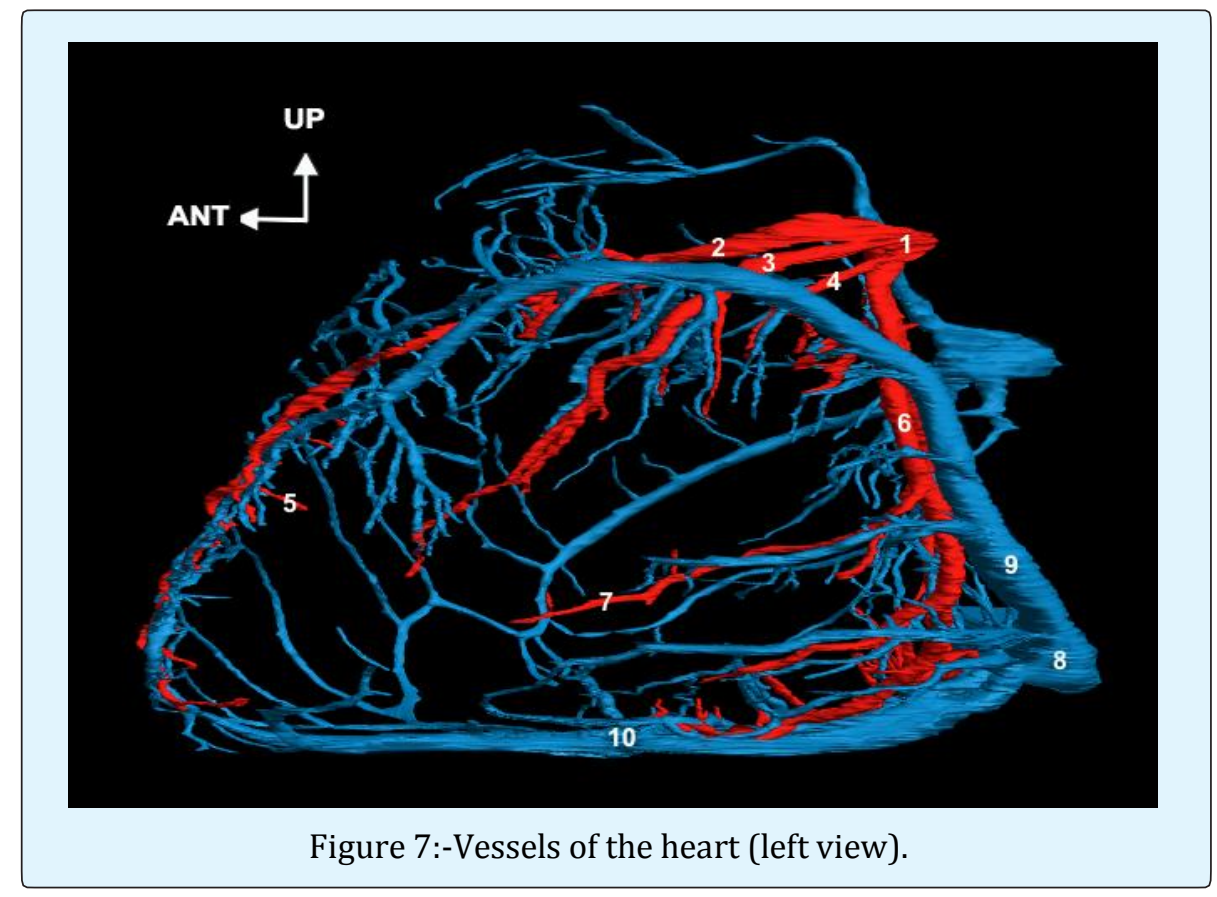

1-left coronary artery (common trunk); 2-anterior interventricular artery; 3-Diagonal artery; 4-"Bissectrice" artery; 5-Septal branch; 6-Circumflex artery; 7-left marginal artery; 8-coronary Sinus; 9-Great cardiac vein; 10-middle cardiac vein; 


\section{Discussion}

There are some limitations of our work:

We did not achieve the reconstruction of the cardiac and thoracic nerves, as well as the lymphatic network and we could improve the 3D models of the vessels, which show some irregularities, related to the Winsurf ${ }^{\circledR}$ software reconstruction.

A similar work was done on the same anatomical slices by the Korean team without 3D modeling of the cardiac venous network. It is downloadable on the website at: www.kvh/ajou/kr. Please notice that the 3D vectorial models of the Korean man slices were also recently used in the Anatomage Table ${ }^{\mathrm{TM}}$ (Copyright $2005^{\odot}$ Anatomage, USA), but they are mostly anatomical models created by computer graphists, and not 3D models outlined directly on the slices.

Future studies are in progress using the whole anatomical slices: virtual dissection table based on modified Acrobat ${ }^{\circledR}$ interface (www.diva3d.com) and another by the Korean team is a virtual percutaneous coronary intervention.

These new computer tools could be developed in the frame of a partnership which is available through the UNESCO chair of digital anatomy, created in Paris Descartes University 3 years ago. (www.anatomieunesco.org)

The aim of this Unesco Chair is to promote the new educational tools of anatomy.

It is also to setup a worldwide network of experts in morphology willing to develop these digital tools. A worldwide partnership is the best mean to setup a huge databank of 3D vectorial models of anatomy validated by experts. In addition to research and educational anatomy, they could be used in other fields, in particular surgical training, simulation and planning.

\section{Conclusion}

By producing these 3D anatomical models, our goal is to provide anatomical data and processing systems that contribute to the education for basic and clinical medicine. The applicability of the sectioned images was also demonstrated in a browsing software [9] developed to freely explore segmented slices and to provide the names of structures, for teaching and learning anatomy, including the liver segmentation $[20,21]$.

In addition, the 3D vectorial models can be exported in a 3D PDF file and explored using the powerful Adobe Acrobat interface. This easily provides a virtual dissection table for the students, and could also be used to simulate surgical operations and training. A large partnership through the Unesco Chair of Digital anatomy should contribute to develop these promising tools in a near future. None of the authors have a conflict of interest.

\section{References}

1. Ackerman MJ (1999) The Visible Human project. A resource for education. Acad Med 74(6): 667-670.

2. Spitzer V, Whitlock D (1998) The Visible Human dataset. The anatomical platform for human simulation. Anat Rec 253(2): 49-57.

3. Park JS, Chung MS, Hwang SB, Lee YS, Har DH, et al. (2005) Visible Korean Human: Improved serially sectioned images of the entire body. IEEE Trans Med Imaging 24(3): 352-360.

4. Park JS, Chung MS, Shin DS, Dong HH, Zang HC, et al. (2009) Sectioned images of the cadaver head including the brain and correspondences with ultrahigh field 7.0 T MRIs. Proc IEEE 97(12): 19881996.

5. Park HS, Choi DH, Park JS (2015) Improved sectioned images and surface models of the whole female body. Int J Morphol 33(4): 1323-1332.

6. Zhang SX, Heng PA, Liu ZJ (2006) Chinese Visible Human Project. Clin Anat 19(3): 204-215.

7. Tang L, Chung MS, Liu Q Shin DS (2010) Advanced features of whole body sectioned images: Virtual Chinese Human. Clin Anat 23(5): 523-529.

8. Yuan Y, Qi LN, Luo SQ (2008) The reconstruction and application of Virtual Chinese Human female. Comput Methods Programs Biomed 92(3): 249-256.

9. Shin DS, Chung MS, Park HS, Park JS, Hwang SB (2011) Browsing software of the Visible Korean data used for teaching sectional anatomy. Anat Sci Educ 4(6): 327-332.

10. Shin DS, Chung MS, Park JS, Hyung SP, Sangho L et al. (2012) Portable Document Format File Showing the Surface Models of Cadaver Whole Body. J Korean Med Sci 27(8): 849-856.

11. Chung BS, Shin DS, Brown P, Choi J, Chung MS (2015) Virtual dissection table including the Visible Korean images, complemented by free software of the same data. Int J Morphol 33(2): 440-445. 
12. Shin DS, Kim HJ, Kim BC (2015) Sectioned Images and Surface Models of a Cadaver for Understanding the Dorsalis Pedis Flap. J Craniofacial Surgery 26(5): 1656-1659.

13. Ros M, Trives JV, Lonjon N (2017) From stereoscopic recording to virtual reality headsets: designing a new way to learn surgery. Neurochirurgie 63(1): 1-5.

14. Park JS, Chung MS, Hwang SB, Lee YS, Har DH, et al. (2005) Technical report on semiautomatic segmentation by using the Adobe Photoshop. J Digit Imaging 18(4): 333-343.

15. Deptuch J (1991) Implementing Boissonnat's approach for generating surface models of craniofacial cartilages. Anat Rec 229(4): 556-564.

16. Lozanoff S, Diewert VM (1989) A computer graphics program for measuring two- and three dimensional form change in developing craniofacial cartilages using finite-element methods. Comput Biomed Res 22(1): 63-82.

17. Moody D, Lozanoff S (1997) SURF driver: a practical computer program or generating 3D models of anatomical structures. Honolulu, Hawaii: 14th Annual Meeting of the American Association of Clinical Anatomists.

18. Uhl JF, Park JS, Chung MS, Delmas V (2006) Threedimensional reconstruction of urogenital tract from Visible Korean Human. Anat Rec A Discov Mol Cell Evol Biol 288(8): 893-99.

19. Shin DS, Park JS, Park HS, Hwang SB, Chung MS (2012) Accessible and informative sectioned images and surface models of a cadaver head. J Craniofac Surg 23(4): 1176-1180.

20. Shin DS, Park JS, Park HS, Hwang SB, Chung MS (2012) Outlining of the detailed structures in sectioned images from Visible Korean. Surg Radiol Anat 34(3): 235-247.

21. Shin DS, Chung MS, Lee JW, Park JS, Chung J, et al. (2009) Advanced surface reconstruction technique to build detailed surface models of liver and neighboring structures from the Visible Korean Human. J Korean Med Sci 24(3): 375-383. 